\title{
Engineering Admission Counseling
}

\author{
Akshay Mantri ${ }^{1}$, Naina SRohra ${ }^{2}$, Naman Verma ${ }^{3}$, Nikita Gaikwad ${ }^{4}$, Prof. Sachin Godse ${ }^{5}$ \\ BE Student, Department of Computer Engineering, Sinhgad Academy of Engineering, Pune, India ${ }^{1,2,3,4}$ \\ Faculty of Computer Engineering, Sinhgad Academy of Engineering, Pune, India ${ }^{5}$
}

\begin{abstract}
Education is indispensible for human. Without education one cannot survive in this world. Every year many student land up in wrong colleges because of lack of information. If a system be devised which can provide them proper college information then a number of students taking admission in wrong colleges can be minimize. This system will also provide prediction for the next or future year cutoff list.
\end{abstract}

Keywords: Admission Process, Admission Counseling, Ant Algorithm, Web Portal for Engineering Admission, Linear Regression

\section{INTRODUCTION}

The Purpose of this document is to describe the match the student's profile and the suitable track channels Engineering admission counseling System which will be used to automate the student admission process and other forms that the student submits for a particular request by introducing the concept of artificial intelligence in the existing system. In this attention has been given to the psychological aspects of the process-especially the accompanying stress and confusion experienced during the decision making process. This system will be providing students who want to take admissions through cap rounds, a Cap round list will be generated according to their preferences of hostel, mess, placement criteria etc. In this module emails will be send to students who have registered their names, for advertisement of mess, hostel, coaching classes and mail will also be send for verification of accounts. Cap round list generated can be converted to PDF, EXCEL, TXT format and print out can also be taken out. After taking admission students get so much trouble while searching for nearer hotels if they are not localities, mess \& hostel for students who want to get admission in hostel. System will also provide the facility of finding nearer bus-stands to go somewhere and it will also provide which buses go to famous places. We will also provide what are the famous places in the city. This system will also provide students, route to go to particular college through various sources. It also provides time table for PMT buses, approximate auto fare.

\section{LITERATURE SURVEY}

The system first analyzes student's academic merit, student records, and the college admission criteria. Then, it predicts the likelihood university college that a student may enter. This software presents a new college admission system based on the data mining techniques and knowledge discovery rules, for tackling college admissions prediction quickly and fairly. If the student does not get the admission in the desired branch of engineering, then they find it difficult to take decision which will be the suitable branch for them. The proposed knowledge base decision technique will guide the student for admission in proper branch of engineering. In addition to the high prediction accuracy rate, flexibility is an advantage, as the system can predict suitable colleges that through which the students are advice to enter.

\section{EXISTING SYSTEM}

The current system consists of mock round in which students come to know about the colleges in which he/she cannot get admission. So in the first round the student does not enter into those colleges. Basically, the mock round introduced in the current DTE system is about rejecting the colleges in which the students will not get the admission. There are more three rounds in the admission process but still people are not satisfied with the results at the end.

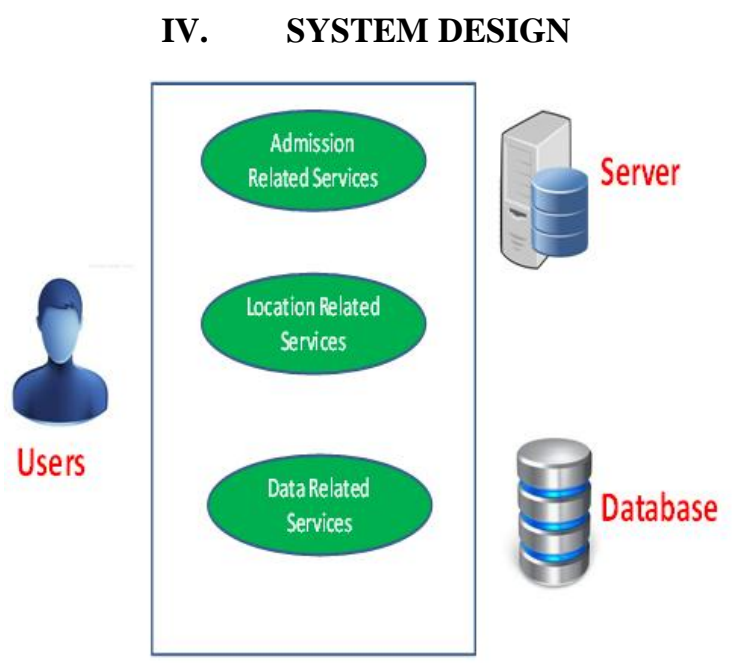

Figure no 1 system model

The proposed system is designed to make students life simple. There are three types of active users namely Student, College, Proprietor. All can register into system and use the system features.

Students (him or her) can register in this model. After that there is one module which will ask his or her qualification, entrance exam score and preferences (hostel, mess, branch) then system will generate CAP list containing the name of colleges, student can get. List contains the name 
of suggested college. If student is not satisfied with the college then he or she can go for CAP round 2 or round 3. Once student got the college then student can see the path from his/her home to respective college, after that student user can see nearby places of college. Second user will be college. College will register into the system and provide info related to college like about mess, hostel, transportation facilities, whether it is girls college or not etc. College can also provide information about the events held in their college. Third user will be proprietor who can register himself and provide information regarding his/her services.

\section{MODULES}

\section{A. Registration Process}

There are three type of user namely student, college, proprietor. All can register into system. There will be one interface from where they can register themselves. After registration they all can enjoy the system features. In student, there are three types namely Admission User, Admitted User and Normal User. All can register in this portal via filling the registration form.

\section{B. Email Sending}

After registration, an email will be send to email id of respective user for verification purpose. Once the email sent to the user, he/she has been verified. An OTP(one time password) will be generated and send to the email id of respective user, user need to enter that OTP and proceed for further functions.

\section{Find your interest}

There will be one section in which user of student type can find their interest by giving one online test. After evaluation of exam, student gets its result in the form of branch percentage. There will be 20 questions of all branches. Questions will come randomly and each option corresponds to tow or more branches. After the exam, result will be in the form of percentile format.

\section{Cut off marks Prediction}

In this module we will use data mining technique to predict the next year's cut off list based on the previous year's two or three year's cutoff list. We will use linear regression to predict the marks of next year.

\section{E. Around Me Facilities}

In this module, student will enter the college name and our system will show him/her the whole map containing college's address, nearby mess, hostel, hospitals, medical stores, stationary, ATM. This all will be stored in database as they are registered user of our portal. We will retrieved this from database and show it on map.

\section{F. $\quad$ Route Finding}

Student will enter source address and destination address, our system will show the path on map and also calculate the distance (in $\mathrm{km}$ ) from source to destination. System will also generate the charges (in rupees) from source to destination.

\section{IMPLEMENTATION DETAIL}

Prediction Module

1) Take the college name, branch name and cap round no for which the cutoff is to be predicted.

2) Find all the previous scores for that college branch and cap round no from the cutoff table.

3) Save year of establishment, CET, AIEEE and diploma score in separate arrays.

4) Find total of each array.

5) Find mean for each array ie. divide the total sum of array with the no. of previous year cutoffs referred.

6) Follow this formula

7) For(no of previous yr cutoff referred) \{

7.1) $\mathrm{x}=[(\mathrm{yrs}$ of est - mean yrs of establishment $) *($ cutoff

for that yrs of establishment - mean cuttoff $)]^{*} \mathrm{x}$

7.2) $y=y+$

(yrs of establishment - mean yrs of establishment $)^{\wedge} 2$ \}

8) $W 1=x / y$

9) $\mathrm{W} 0=$ cutoff mean $-($ yrs of establishment mean $)(w 1)$

10) Cuttoff $=w 0+w 1 *(y r s$ of establishment $)$

11) End

B. Find Your Interest Module

1) Retrieve Question(str)

The format of str will be Option Count\# Question \# Option1 \# Option2\# Option3\# Option4 \#Option5 \#

1) Declare que,p1,p2,p3,p4,p5,opt[]

2) Set $\mathrm{p} 1$ to 0

3) Set $\mathrm{p} 2$ to 2

4) Set $\mathrm{p} 3$ to position of \# after p2+1

5) Set $\mathrm{p} 4$ to position of \# after p3+1

6) Set $\mathrm{p} 5$ to position of \# after p4+1

7) Set $\mathrm{p} 6$ to position of \# after p5+1

8) Set $\mathrm{p} 7$ to position of \# after p6+1।

9) Set optcount to character at position 1 of str

10) Set que to substring from position 2 to $p 2$

11) Set opt[0] to substring from position $p 2+1$ to $p 3$

12) Set opt[1] to substring from position $p 3+1$ to $p 4$

13) Set opt[2] to substring from position $\mathrm{p} 4+1$ to $\mathrm{p} 5$

14) Set opt[3] to substring from position $\mathrm{p} 5+1$ to $\mathrm{p} 6$

15) Set opt[4] to substring from position $\mathrm{p} 6+1$ to $\mathrm{p} 7$

1) For $i=0, j=1$ to optcount

1.1. Create the radio button with value Option+' $\mathrm{j}$ '

i. GenerateQueNo() ran_que = Math.floor $(($ Math.random ()$* 20)+1)$ search ran_que in visited_que[] if Not found insertran_queinvisited_que[]

2) SendQueNo(ran_que) ran_que is generated by GenerateQueNo() and not visited yet.

1) Send ran_que to server.

2) Get response from Server.

3) Call RetrieveQuestion(response).

4) Set flag to 0 . 


\section{International Journal of Advanced Research in Computer and Communication Engineering}

Vol. 4, Issue 5, May 2015

3) InitQuiz()

1) Initialize qno to 1 and flag to 0

2) Call GenerateQueNo()

3) Call SendQueNo()

4) SaveResult(ran_que,res)

1) Insert ran_que in que_list[].

2) Insert res into ans_list[].

5) Send Answer()

1) Declare q,a,brlist[].

2) Set q to que_list

3) Set a to ans_list

4) Send q and a to Server.

5) Get the response from Server.

6) Parse the JSON response.

7) Display the result.

\section{SYSTEM FEATURES}

1) Immediate Feedback: The System must try to answer all the queries of the students and it should provide immediate feedback after any request from the students.

2) Reduce the cost of Admission Process: The main aim of the System is to reduce the cost needed for Admission Process, so it automatically reduces the manual power needed to perform the entire task and improve the quality of the work.

3) Make the interface Simple as Possible: The System must provide the simple and easy interface for beginners and also provide facilities for technical people who are using the system.

4) Reduced time: To perform any task time is one of the important factors to consider. If the system not utilized properly time, than the entire aim of system fails and the system fails to reach its goal.

5) Reach to geographically scattered students: One of the important objectives of the system is communicate with all the students scattered geographically.

6) Centralized data handling: Transfer the data smoothly to all the departments involved and handle the data centralized way.

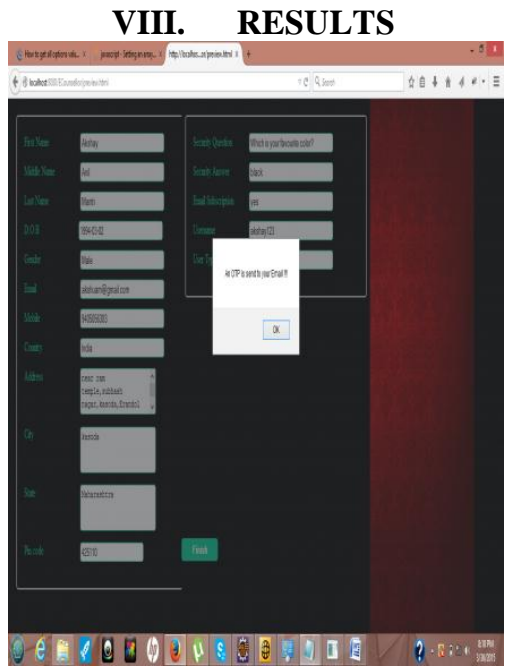

Figure 2 Email Sending for verification

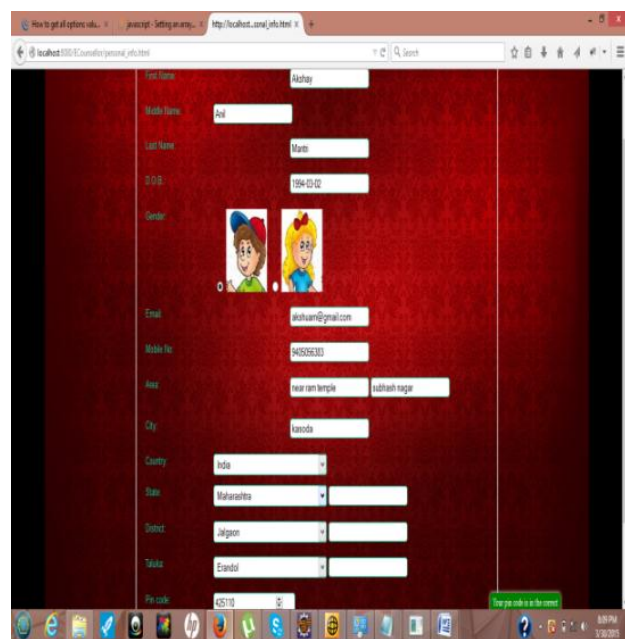

Figure 3 Admission User Registrations

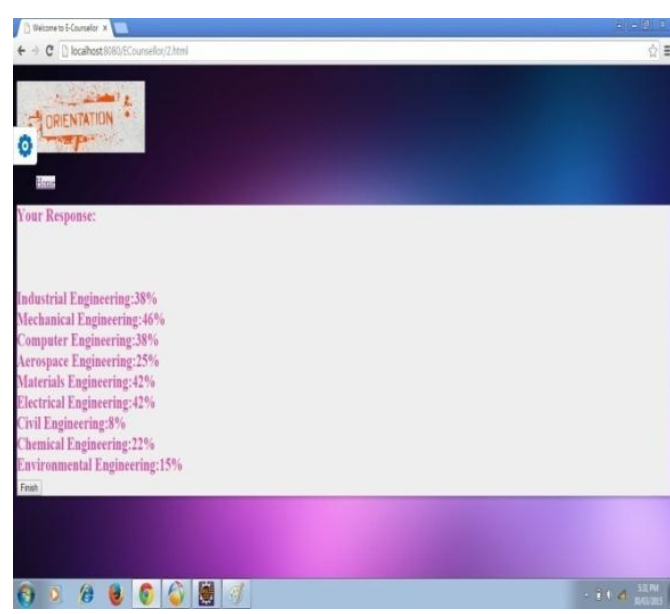

Figure 4 Quiz Results

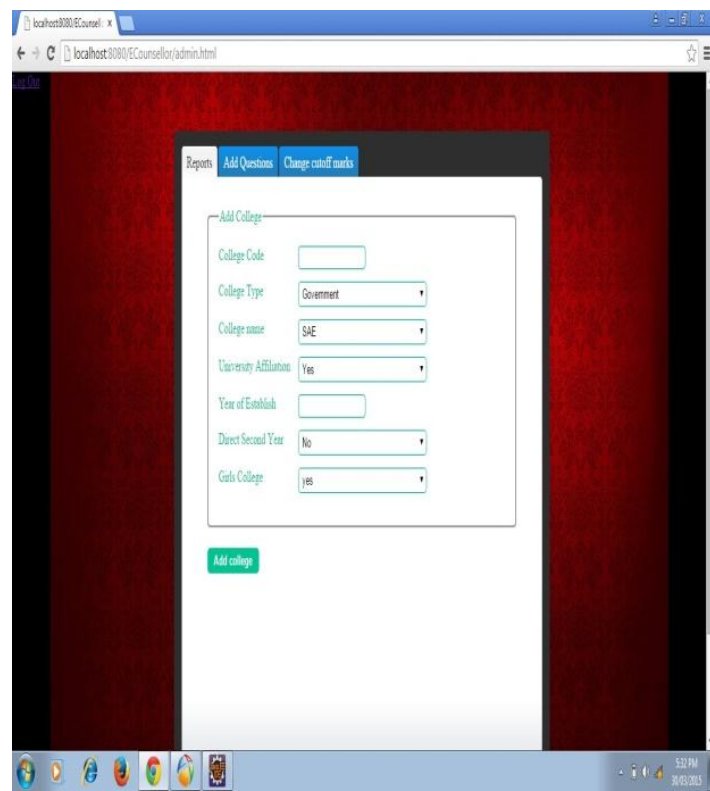

Figure 5 Admin

\section{CONCLUSION}

Admissions to engineering colleges in the Maharashtra state is based upon the score in common entrance test (CET) and those who are not from Maharashtra state is 
based on All India entrance exam (AIEEE). Many Students get confused while filling the options form. So our system will reduces the stress of students while filling the options form. On one click of button student will get list of possible colleges in which they can get the admission and it will be easy for them to give preferences.

\section{ACKNOWLEDGEMENT}

This project would not have been possible without the kind support and help of many individuals. We would like to extend our sincere thanks to all of them. First, we would like to express our sincere gratitude to our project guide Prof. S. P. Godse, whose valuable guidance, encouragement, and provision of necessary facilities made this work possible. We are thankful to our Head of Department Prof. B. B. Gite whose help and knowledge was the support to complete our project. Finally our great thanks to our industry guide Mr. Rupesh Pawar whose valuable guidance and encouragement made this work possible.

\section{REFERENCES}

[1]. Sangari.R.S, Dr.M.Balamurugan"A Survey on Linear Regression Using Data Mining " ,International Journal of Computer Science and Mobile Applications on DOI:2,february-2014,Publication Year:2014,Page(s):84-88,vol.2,ISSN:2321-8363

[2]. Linear Regression Analysis ,second edition By George A. F. Seber, Alan J. Lee, ISBN-13: 978-0471415404

[3]. Data Analysis Using Regression and Multilevel/Heirarchical Models, Paperback-December 18,2006 By Andrew Gelman,Jennifer Hill, Edition-1, ISBN-13:978-0521686891

[4]. Java Forums: https://www.java.net/forums

[5]. http://www.w3schools.com/

[6]. http://dev.mysql.com/ 